\title{
CHEMICAL COMPOSITION OF AN EMANATION FROM COMETS: IDENTIFICATION OF THE 3 MICRON COMET FEATURE
}

\author{
A.SAKATA ${ }^{1}$, S.WADA $^{2}$ and A.T.TOKUNAGA \\ 1. Dept. of Applied Phys. and Chem., University of Electro-Communications, \\ Chofugaoka, Chofu, Tokyo 182, Japan \\ 2. Dept. of Chemistry, University of Electro-Communications, Chofugaoka, \\ Chofu, Tokyo 182, Japan \\ 3. Institute for Astronomy, University of Hawaii, 2680 Woodlawn Dr., \\ Honolulu, HI 96822 U.S.A.
}

\begin{abstract}
Recent high resolution observations of comets revealed a detailed spectral shape of the $3.4 \mu \mathrm{m}$ feature. We measured IR spectra of simple 14 hydrocarbon molecules and made "synthesized comet spectrum". Peak wavelength and spectral shape of the synthesized spectrum are well in agreement with the observed comet features.
\end{abstract}

\section{Introduction}

Recent high resolntion observations of comet Halley (1986), comet Wilson (1987), comet Bradfield (1987), comet Brorsen-Metcalf (1989) and comet Okazaki-Levy-Rudenko (1989) showed similar features in the $3 \mu \mathrm{m}$ region. They have a peak at $3.36-3.38 \mu \mathrm{m}$ and small humps near $3.5 \mu \mathrm{m}$. Comet Brorsen-Metcalf and comet Okazaki-Levy-Rudenko showed higher feature-to-continuum ratios at $3.4 \mu \mathrm{m}$ than other comets (Brooke et al., 1990).

We synthesized the $3.4 \mu \mathrm{m}$ feature with IR spectra of gaseous hydrocarbon molecules. The synthesized feature is in good agreement with the observed comet features.

\section{Experiment and Result}

We used a JASCO-810 IR spectrophotometer to obtain IR spectra of 14 hydrocarbon molecules in a specially-designed gas cell. We made a synthesized spectrum from conbinations of each hydrocarbon spectrum, in which the highest peak is normalized as unity. The features at $3 \mu \mathrm{m}$ of molecules are classified into three types as shown in Fig.1.

IR spectra of low-molecular-weight saturated hydrocarbons (hereafter LSHs) containing 2-6 carbon atoms, such as ethane, propane, butane, isobutane, n-pentane, and n-hexane, show a peak near $3.38 \mu \mathrm{m}$. A sum of LSHs spectra is shown in Fig.1 (A). Each of four saturated hydrocarbons containing of 7-10 carbons, such as n-pentane, n-octane, $n$ nonane, and $n$-decane $h$ as a very similar spectrum peaked at $3.42 \mu \mathrm{m}$, accompanying minor peaks at 3.38 and near $3.5 \mu \mathrm{m}$. An IR spectrum synthesized as a sum of spectra of these high-molecular-weight saturated hydrocarbons (hereafter HSHs) is indicated in Fig.1(B). Low molecular weight unsaturated hydrocarbons (hereafter LUHs) containing 2-4carbons, such as ethylene, propylene, butene, and butadiene, show a specific own feature. An IR

241

A.C. Levasseur-Regourd and H. Hasegawa (eds.), Origin and Evolution of Interplanetary Dust, 241-244.

C 1991 Kluwer Academic Publishers, Printed in Japan. 


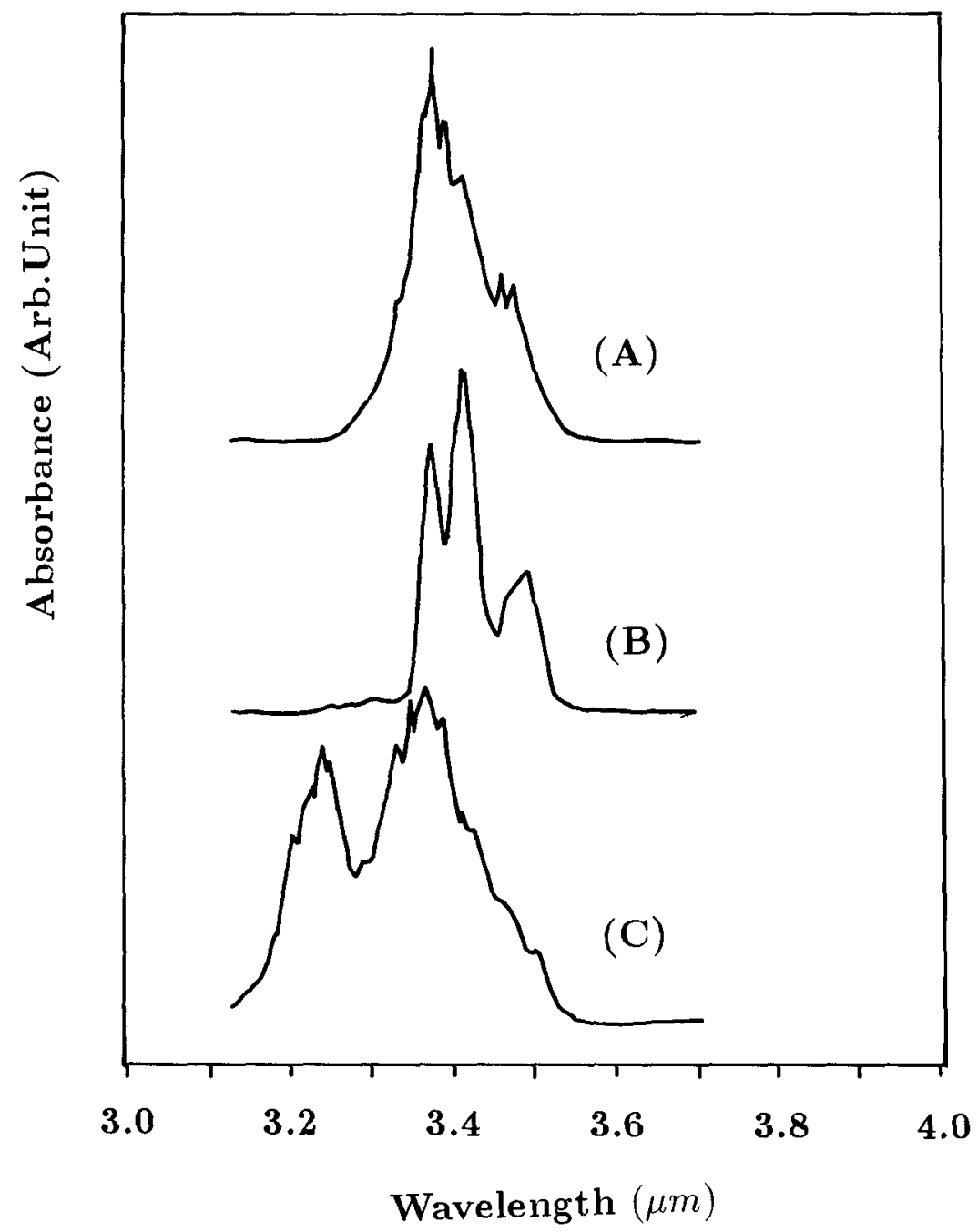

Fig.1, Three synthesized spectra, (A); from spectra of saturated hydrocarbons having 26 carbons, (B); from those having 7-10 carbons, and (C); from spectra of unsaturated hydrocarbons having 2-4 carbons. In the spectrum of each hydrocarbon, the main peak height is normalized as unity. Each synthesized spectrum is made from hydrocarbon spectra in the ratio of $; 1.5$ ethane : 1.5 propane : 1.0 butane : 1.0 iso-butane : 0.5 pentane : 0.5 hexane in (A), 1.0 heptane : 1.0 octane : 1.0 nonane : 1.0 decane in (B), and 1.0 ethylene : 1.0 propylene : 1.0 butene : 1.0 butadiene in $(\mathrm{C})$. 


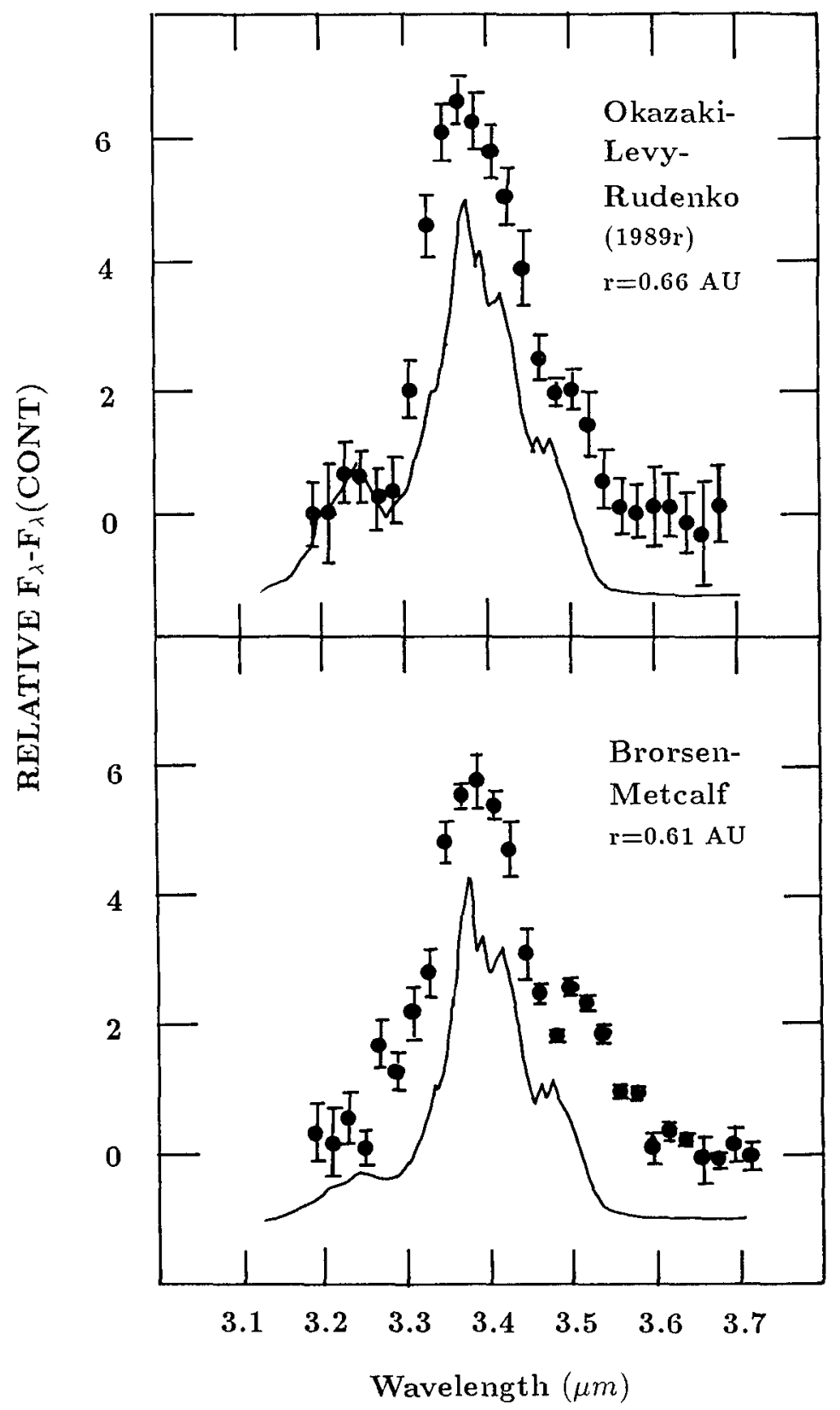

Fig.2. Comparison of "synthesized comet spectra" to the observed comet spectra. Solid lines show "synthesized comet spectra" of hydrocarbons in the ratios of $1.0(\mathrm{~A}): 0.5(\mathrm{~B})$ : $1.5(\mathrm{C})$ in an upper figure and $1.0(\mathrm{~A}): 0.5(\mathrm{~B}): 0.5(\mathrm{C})$ in lower figure. The symbols $(\mathrm{A})$, (B), and (C) are the same used in Fig.1. 
spectrum of LUHs, synthesized in a similar manner, is shown in Fig.1 (C). Its peaks are located at 3.37 and $3.23 \mu \mathrm{m}$.

"Synthesized comet spectra" were calculated from combination of three spectra of LSHs, HSHs, and LUHs spectra in the ratio of $2: 1: 1$ and $2: 1: 2$ and compared to the spectrum of comet BM and comet OLR, respectively.

\section{Discussion}

The intensity of the $3.4 \mu \mathrm{m}$ feature is correlated with the water production rate (Brooke et al., 1990). These facts suggest that the $3.4 \mu \mathrm{m}$ emitter in comets consists of a complex mixture of organic compounds (Knacke et al, 1986), which may be volatile.

We propose that the emitter in comets may be a mixture of hydrocarbon molecules. The peak position of LSHs spectra fits to comet spectra at $3.36-3.38 \mu \mathrm{m}$, but the width is narrower than that of the comet spectra. The addition of HSHs spectra to LSHs spectra broadens the peak in the long wavelength side, while the addition of LUHs spectra causes the broadening of the peak in the short wavelength side. The addition causes also humps near $3.5 \mu \mathrm{m}$ and at $3.2-3.3 \mu \mathrm{m}$, respectively. The abundance ratios of LSHs, HSHs, and LUHs affect the shape of "synthesized comet spectra" as shown in Fig.2.

"Synthesized comet spectra" showed the similar $3.4 \mu \mathrm{m}$ feature to those of comet OLR and comet BM. The main component of the emitter in comets may be low-molecular-weight hydrocarbons. The emitter of comet OLR contains more unsaturated hydrocarbons than that of the comet BM.

We excluded methane from our hydrocarbon mixture, because it shows a strong sharp peak at $3.32 \mu \mathrm{m}$. As it has a lowest boiling point amongst all hydrocarbons, it might behave differently from other hydrocarbon gases in cometary conditions.

\section{Conclusion}

The $3.4 \mu m$ features synthesized with IR spectra of gaseous hydrocarbons agree well with the observed comet spectra.

\section{References}

Brooke, T. Y., and Tokunaga, A. T. (1990)' Detection of the $3.4 \mu \mathrm{m}$ emission feature in comets Brorsen-Metcalf and Okazaki-Levy-Rudenko (1989r) and an observational summary', submitted to A. J.

Knacke, R. F., Brooke, T. Y., and Joyce, R. R. (1986) 'Observations of 3.2-3.6 micron emission features in comet Halley', Ap. J. 310, L49-L53. 